\title{
RESULTS OF COORDINATED MULTIWAVELENGTH OBSERVATIONS OF SOLAR-TYPE STARS
}

\author{
J.HUOVELIN \\ Observatory and Astrophysics Laboratory, University of Helsinki, \\ Tähtitorninmäki, SF-00130 Helsinki, Finland \\ S.H.SAAR \\ Harvard-Smithsonian Center for Astrophysics, Mail Stop 58, \\ 60 Garden Street, Cambridge, MA 02138, USA
}

\begin{abstract}
We present results of nearly simultaneous polarimetric and $\mathrm{Ca} \mathrm{II}(\mathrm{H}+\mathrm{K})$ emission observations taken over a full rotation period for a sample of six solar-type stars. Significant variations are seen for two stars in polarization and four stars in $\mathrm{Ca}$ emission, which we interpret as due to rotational modulation of magnetic areas on the stellar surface. The irregularity of the variations suggest changes of the area size and/or distribution in time scales of the rotation period. Preliminary models (including rough surface maps of two stars) and future prospects are discussed.
\end{abstract}

\section{Introduction and Observations}

Magnetic activity in late-type stars gives rise to a multitude of effects in their atmospheres, modifying the observed radiation throughout the spectrum. At the photospheric level, the Zeeman effect may broaden the profiles of the magnetically sensitive absorption lines, and recently, several techniques have been developed to analyze this phenomenon using the unpolarized stellar spectra (e.g., Robinson, 1980; Saar, 1988; Mathys and Solanki, 1989). The temperature in magnetic region may be greater or smaller than in the surrounding photosphere, causing both photometric and line profile variations as the region cross the stellar disk. Recently, inversion methods ("Doppler" or "surface" imaging) have been developed to solve the temperature distribution of the stellar surface from a time series of spectroscopic observations (Vogt et al., 1987; Piskunov et al., 1989).

At chromospheric level, the ultraviolet emission lines, Ca II H and K, He D3 (5876 $\AA$ ), $\mathrm{H} \alpha$, and the $\mathrm{Ca}$ IR triplet are indicators of activity. Emission in Ca II (Baliunas et al., 1983) and $\mathrm{Mg}$ II (Neff et al., 1989) show convincing evidence of rotational modulation, indicating chromospheric active areas which live longer than one period of rotation.

Chromospherically active late-type stars have also shown indications of variability in broadband linear polarization (Huovelin et al. 1985, 1988, 1989). The variations are likely due to either the effect of variable magnetic field on saturated photospheric absorption lines (i.e. desaturation of perpendicularly polarized Zeeman components), or to Rayleigh scattering in the optically thin layers of the atmosphere, with the former being more probable in solar-like dwarfs (Huovelin et al., 1988). Monitoring several activity indicators simultane- 
ously over a rotational period is an effective way to obtain information of the stellar surface inhomogeneities. First attempts combining simultaneous polarimetry, magnetic field, and Ca-emission observations are reported by Saar et al. (1988) and Huovelin et al. (1988).

In October 1987, we made new observations that include simultaneous polarimetry, $\mathrm{Ca}$ II $\mathrm{H}$ and $\mathrm{K}$, and magnetic field observations for several active solar type dwarfs. The polarimetric observations were made at the Crimean Astrophysical Observatory between October 2 and 14, 1987. The instrument was a five channel (UBVRI) version of the photopolarimeter of the University of Helsinki (Piirola 1973, 1975) connected to the 1.25 meter AZT-11 telescope of the Crimean Observatory. The Ca II $\mathrm{H}$ and $\mathrm{K}$ line emission observations were obtained contemporaneously with the four-channel photoelectric spectrometer at the Cassegrain focus of the Mt Wilson 1.5 meter telescope, and reduced as described by Vaughan, Preston and Wilson (1978). The S-index values were converted to chromospheric surface fluxes $\mathrm{F}_{H K}^{\prime}$ using the formulae in Huovelin et al. (1988). The high resolution spectroscopic observations (617 $\mathrm{nm}$ region) for the magnetic field and filling factor determinations were made with the National Solar Observatory McMath echelle/CCD system. We summarize some preliminary results of the analysis of the polarimetric and $\mathrm{Ca}$ II observations in this paper.

\section{Results and Discussion}

For several stars, the overall variations during the observing period were small and fairly irregular. We, therefore, first studied whether statistical fluctuations could be the cause of these changes. As the method we applied a standard $\chi^{2}$ test (see e.g. Huovelin et al., 1989). The results of this test are shown in Table 1.

Table 1: Programme stars and statistical results. $P_{\text {rot }}$ values are from Noyes et al. (1984).

\begin{tabular}{|c|c|c|c|c|c|c|c|c|}
\hline HD no. & Name & Sp. type & $\overline{B-V}$ & $\begin{array}{c}P_{\text {rot }} \\
\text { (days) }\end{array}$ & $\begin{array}{c}\text { significance } \\
P(U)\end{array}$ & $\begin{array}{c}\text { of } \\
P(B)\end{array}$ & $\begin{array}{c}\text { variations } \\
P(V)\end{array}$ & $\begin{array}{c}(\%) \\
\text { Ca-em. }\end{array}$ \\
\hline 1835 & 9 Cet & G2V & 0.66 & 7.7 & $\geq 99.99$ & 96.3 & 88.8 & 92.3 \\
\hline 17925 & & K0V & 0.87 & 6.6 & 85.5 & 92.8 & 76.8 & $\geq 99.99$ \\
\hline 20630 & $\kappa$ Cet & G5V & 0.68 & 9.4 & 99.6 & 89.4 & 71.2 & $\geq 99.99$ \\
\hline 22049 & $\epsilon$ Eri & $\mathrm{K} 2 \mathrm{~V}$ & 0.88 & 11.3 & 99.4 & 99.98 & 96.0 & $\geq 99.99$ \\
\hline 39587 & $\chi^{1}$ Ori & G0V & 0.59 & 5.2 & 84.9 & 97.9 & 92.9 & $\geq 99.99$ \\
\hline 206860 & HN Peg & G0V & 0.59 & 4.7 & 52.1 & 68.4 & 99.1 & 87.4 \\
\hline
\end{tabular}

We note that polarization in the ultraviolet may not always be the best indicator of variations, for although the polarization amplitude is generally the largest in $U$, the observational uncertainties are larger as well, reducing the statistical significance of the detection.

We are developing a procedure to model activity variations, which includes simultaneous calculation of Ca-emission, photometric brightness, linear polarization, and the magnetic 
field as a function of rotational phase. The surface can be covered with arbitrary number of circular spots of arbitrary size and surface brightness. At present, the Ca-emission is assumed to be confined to the magnetic areas, with a uniform intrinsic intensity, and the surface outside the magnetic regions is assumed to produce no emission. For limb darkening we use a linear law $(1-\epsilon+\epsilon \mu)$ with $\epsilon=0.5$ (Schrijver et al., 1989). The current model for the Ca-emission does not yield absolute sizes of the active areas, since the actual levels of emission in the quiet and active areas is unknown (due to unknown and variable contributions from network, spots etc.).
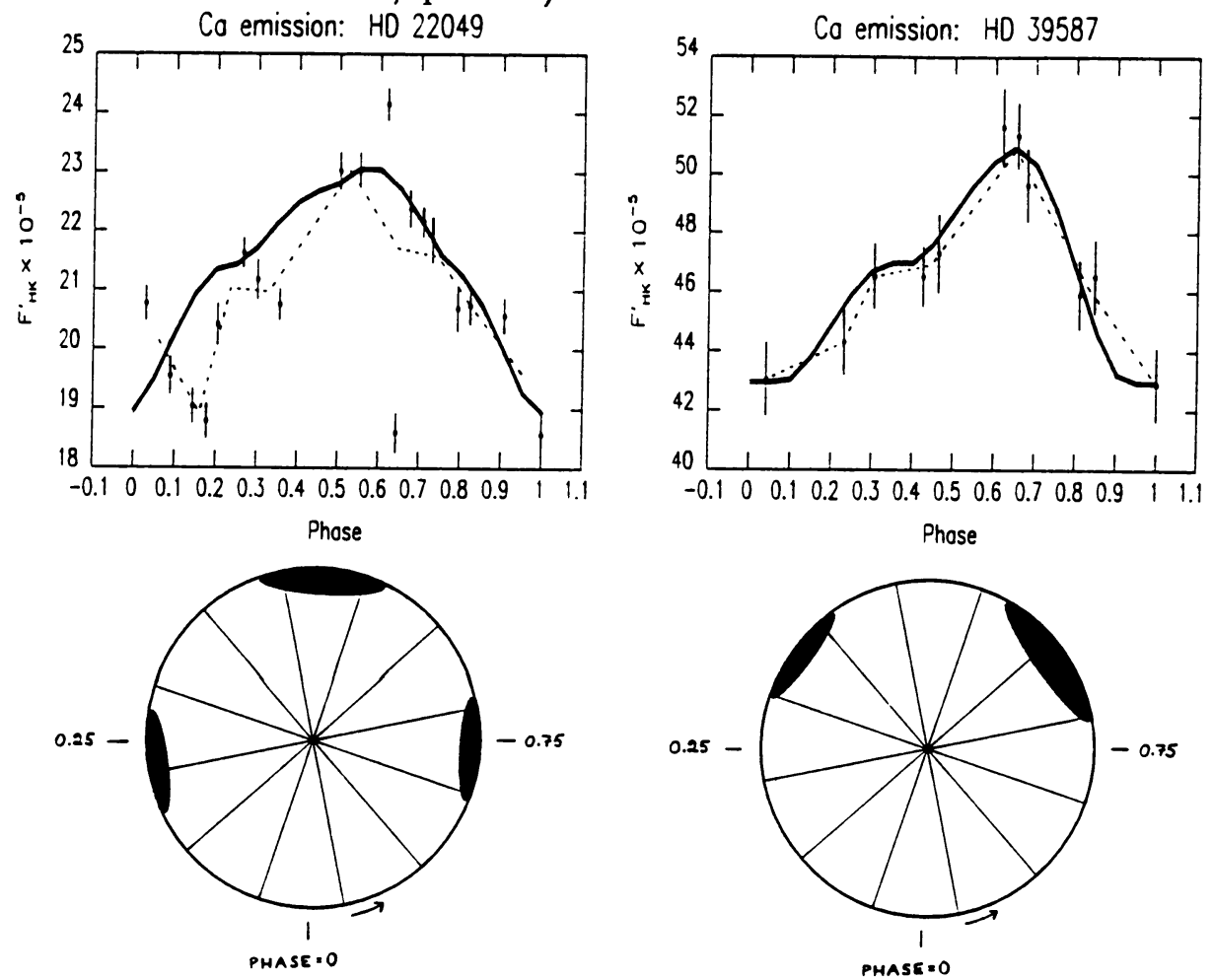

Figure 1. $\mathrm{CaII}(\mathrm{H}+\mathrm{K})$ flux variations $\left(e \mathrm{em}^{-2} \mathrm{~s}^{-1}\right)$ vs. rotation phase and corresponding models (thick lines), which are sketched in longitude maps below. Error bars are $1 \sigma$.

Modeling the Ca II data, we obtain rough estimates for the plage longitudes and an upper limit for their relative sizes. In Fig.1 we present the preliminary interpretation of the Ca-emission variations in $\epsilon$ Eridani with three and $\chi^{1}$ Orionis with two equatorial active regions. The plage sizes are exaggerated for visibility. Future work includes the interpretation of simultaneous polarimetry and magnetic field measurements together with Ca-emission, using more detailed models described below.

The polarimetric model is based on differential saturation of magnetically sensitive absorption lines (i.e. magnetic intensification). The basic scheme is an improved version of the Landi Degl'Innocenti (1982) model, employing explicit disk-integration and improved 
treatment of limb darkening (Saar and Huovelin, 1989 in preparation). For comparison, we can also produce models of the polarization variations assuming Rayleigh scattering as the cause of the polarization. The magnetic field model is the extension of the analysis method of Saar (1988) to inhomogeneous stars, adding the filling factors from the active regions with background fields to form disk-integrated line profiles.

By fitting the combined theoretical model (Ca-emission, linear polarization and magnetic field) to the observations with a suitable inversion method (e.g., Piskunov et al., 1989), we can obtain estimates for the coordinates, sizes and magnetic fields of the active areas, and also for the stellar inclination. From the polarimetry, it is, in principle, also possible to derive the orientation of the stellar rotation axis in the sky. With the present data, however, the surface brightnesses of the active areas remain ambiguous due to lack of simultaneous photometry. Since the current combination of observations should be fairly insensitive to areas considerably cooler than the surrounding photosphere, a reasonable first approximation might be to assume that the active areas and the surrounding photosphere are equally bright. The models can be refined using future simultaneous observations with photometry included.

Acknowledgements. This work has been supported by a grant from the Aaltonen Foundation (JH) and by the Smithsonian Institution postdoctoral research fellowship program (SS). We are indebted to Dr. S. Baliunas for providing the Ca II data.

\section{References}

Baliunas, S., Vaughan, A., Hartmann, L., Middelkoop, F., Mihalas, O., Noyes, R., Preston, G., Frazer, J., Lanning, H.: 1983, Astrophys. J., 275, 752

Landi Degl'Innocenti, E.: 1982, Astron. Astrophys., 110, 25

Huovelin, J., Linnaluoto, S., Piirola, V., Tuominen, I., Virtanen, H.: 1985, Astron. Astrophys., 152, 357

Huovelin, J., Saar, S., Tuominen, I.: 1988, Astrophys. J., 329, 882

Huovelin, J., Linnaluoto, S., Tuominen, I., Virtanen, H.: 1989, Astron. Astrophys. Suppl. Ser., (in press)

Mathys, G., Solanki, S.K.: 1989, Astron. Astrophys., 208, 189

Neff, J.E., Walter, F.M., Rodonó, M., Linsky, J.L.: 1989, Astron. Astrophys., 215, 79

Noyes, R.W., Hartmann, L., Baliunas, S., Duncan, D., Vaughan, A.: 1984, Astrophys. J. , 279, 763

Piirola, V.: 1973, Astron. Astrophys., 27, 383

Piirola, V.:1975, Ann. Acad. Sci. Fenn., A VI, No. 418

Piskunov, N.E., Tuominen, I., Vilhu, O.: 1989, Astron. Astrophys., submitted

Robinson, R.D.: 1980, Astrophys. J., 239, 961

Saar, S.H.: 1988, Astrophys. J., 324, 441

Saar, S.H., Huovelin, J., Giampapa, M.S., Linsky, J.L., Jordan, C.: 1988, in Activity in Cool Star Envelopes, Eds. O.Havnes et al., Kluwer, Dordrecht, p.45

Schrijver, C., Coté, J., Zwaan, C., Saar, S.H.: 1989, Astrophys. J., 337, 964

Vaughan, A.H., Preston, G. W., Wilson, O. C.: 1978, Publ. Astron. Soc. Pacific, 90, 267

Vogt, S.S., Penrod, G.D., Hatzes, A.P.: 1987, Astrophys. J., 321, 496 